\title{
A Full-Potential Local-Orbital Approach to the Density-Functional Calculations of Solids
}

\author{
Shugo SuZUKI* and Kenji NAKAO \\ Institute of Materials Science, University of Tsukuba, Tsukuba 305
}

(Received July 3, 1997 )

\begin{abstract}
A full-potential linear-combination-of-atomic-orbitals method based on the density-functional theory developed in the field of the molecular science is extended to the density-functional calculations of solids. It is shown that the method is also useful for studying both the structural and electronic properties of solids. The interatomic distances of graphite and hexagonal boron nitride are calculated within the error of less than $1 \%$. Also, the atomization energies and the elastic constants are properly reproduced by the present method.
\end{abstract}

KEYWORDS: full-potential calculations, LCAO method, density-functional theory, band calculations, structure optimizations

\section{§1. Introduction}

The structural and electronic properties of solids have been studied on the basis of the densityfunctional theory. ${ }^{1,2)}$ There are several methods of the density-functional calculations of solids; the ab initio pseudopotential method with plain waves (PP-PW), ${ }^{3)}$ the augmented-plane-wave method $(\mathrm{APW}),{ }^{4)}$ the Korringa-Kohn-Rostocker Green's function method, ${ }^{5)}$ the linear-muffin-tin-orbital method, ${ }^{6)}$ and the linear-combination-of-atomic-orbitals (LCAO) method. ${ }^{7-9)}$ One important feature is that these methods of the recent improved versions employ the full-potential approach. That is, no shape approximations are introduced in the calculations of the electrostatic potential. This is primarily indispensable for the structure optimization.

Among the extensive studies of solids performed by the above methods, there are only a few studies of the full-potential calculations by the LCAO method, especially of the structure optimization. $\left.{ }^{8}{ }^{9}\right)$ This is due to the difficulty of the full-potential calculations in the LCAO scheme as well as due to the difficulty of the numerical treatment of the three-dimensional multicenter integration in the real space.

On the other hand, the full-potential LCAO method has been successfully applied to the studies of both the structural and electronic properties of molecules. ${ }^{10-25)}$ In the last two decades, powerful

\footnotetext{
*E-mail: shugo@ims.tsukuba.ac.jp
} 
techniques have been developed to the LCAO method of molecules: the efficient method of the total-energy calculations, ${ }^{11,26)}$ the atomic partitioning method of the three-dimensional numerical integration, ${ }^{19,20,22,27-29)}$ the method of solving the Poisson equation, ${ }^{22,30)}$ etc. The extension of these methods for solids is important to the study of solids by the LCAO method. That is, the full-potential local-orbital approach developed in the molecular LCAO methods is expected to be also useful for performing the density-functional calculations of solids.

The purpose of the present study is to extend the full-potential local-orbital approach developed in the molecular LCAO methods to the density-functional calculations of solids. We show that this approach is also useful for studying both the structural and electronic properties of solids.

\section{$\S 2 . \quad$ Methodology}

The density-functional calculations of a solid are performed by solving the following Kohn-Sham equations in a self-consistent way. ${ }^{2)}$

$$
\begin{gathered}
{\left[-\frac{1}{2} \triangle+v_{e s}(\boldsymbol{r})+v_{x c}^{\sigma}(\boldsymbol{r})\right] \psi_{n \boldsymbol{k}}^{\sigma}(\boldsymbol{r})=\varepsilon_{n \boldsymbol{k}^{\sigma}}^{\sigma} \psi_{n \boldsymbol{k}}^{\sigma}(\boldsymbol{r}),} \\
n_{e}(\boldsymbol{r})=\sum_{\sigma} n_{e}^{\sigma}(\boldsymbol{r}) \\
n_{e}^{\sigma}(\boldsymbol{r})=\frac{1}{N} \sum_{n \boldsymbol{k}} f_{n \boldsymbol{k}}^{\sigma}\left|\psi_{n \boldsymbol{k}}^{\sigma}(\boldsymbol{r})\right|^{2} .
\end{gathered}
$$

Here, $\sigma$ denotes the spin of the electrons, $\uparrow$ and $\downarrow$. In addition to the spin quantum number, the oneelectron wave function, $\psi_{n \boldsymbol{k}}^{\sigma}(\boldsymbol{r})$, has two more quantum numbers, the band index $n$ and the wave vector $\boldsymbol{k}$. The sum of $\boldsymbol{k}$ is performed over the Brillouin zone (BZ); the total number of $\boldsymbol{k}$, represented by $N$, is equal to the total number of the unit cells in the whole solid, provided that the periodic boundary condition is used. In eq. (1), $v_{e s}(\boldsymbol{r})$ is the electrostatic potential due to the nucleus and the electron charge densities, $n_{n}(\boldsymbol{r})$ and $n_{e}(\boldsymbol{r})$. Also, $v_{x c}^{\sigma}(\boldsymbol{r})$ is the exchange-correlation potential for the $\sigma$-spin electrons; in the present study, we use the local-spin-density-approximation (LSDA) potential expressed by the Perdew-Zunger parameterization of Ceperley and Alder results. ${ }^{41,42)}$ In eq. (2), $n_{e}(\boldsymbol{r})$ consists of the up-spin and the down-spin contributions, $n_{e}^{\uparrow}(\boldsymbol{r})$ and $n_{e}^{\downarrow}(\boldsymbol{r})$. These electron charge densities are given by using $\psi_{n \boldsymbol{k}}^{\sigma}(\boldsymbol{r})$ and the occupation number of the level $n \boldsymbol{k} \sigma$, $f_{n \boldsymbol{k}}^{\sigma}$, provided that $\psi_{n \boldsymbol{k}}^{\sigma}(\boldsymbol{r})$ is normalized in the unit cell.

First, we describe the method for solving eq. (1). Given $v_{e s}(\boldsymbol{r})$ and $v_{x c}^{\sigma}(\boldsymbol{r})$, we solve eq. (1) by expanding $\psi_{n \boldsymbol{k}}^{\sigma}(\boldsymbol{r})$ by basis functions as follows.

$$
\begin{aligned}
& \psi_{n \boldsymbol{k}}^{\sigma}(\boldsymbol{r})=\sum_{p} C_{p n \boldsymbol{k}}^{\sigma} \chi_{p} \boldsymbol{k}^{(\boldsymbol{r})} \\
& \chi_{p \boldsymbol{k}^{(\boldsymbol{r})}}=\sum_{u} \exp \left(\mathrm{i} \boldsymbol{k} \cdot \boldsymbol{R}_{u}\right) \chi_{p}\left(\boldsymbol{r}-\boldsymbol{d}_{p}-\boldsymbol{R}_{u}\right) .
\end{aligned}
$$


Here, $\chi_{p}(\boldsymbol{r})$ is the $p$-th atomic orbital in the unit cell and $\boldsymbol{d}_{p}+\boldsymbol{R}_{u}$ represents its position vector in the $u$-th unit cell. In the present study, we use the numerical-type orbitals as $\chi_{p}(\boldsymbol{r})$ obtained by solving the Kohn-Sham equations of the atoms. The merit of this type of atomic orbitals is the perfect description of the dissociated limit of the constituent atoms within LSDA. This allows us to calculate atomization energies accurately. The expansion (3) results in the following generalized eigenvalue problem:

$$
\sum_{q} H_{p q \boldsymbol{k}}^{\sigma} C_{q n \boldsymbol{k}}^{\sigma}=\varepsilon_{n \boldsymbol{k}}^{\sigma} \sum_{q} S_{p q \boldsymbol{k}} C_{q n \boldsymbol{k}}^{\sigma}
$$

The Hamiltonian and the overlap matrices are given by

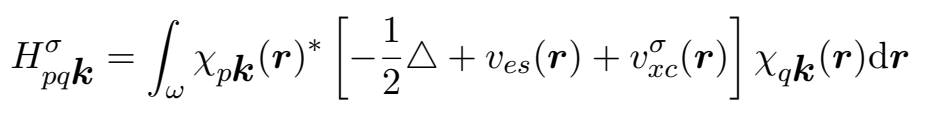

and

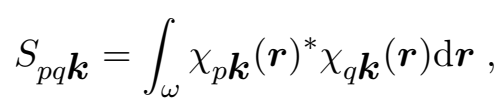

respectively. Here, the integrals over the unit cell are indicated by $\omega$. The three-dimensional numerical integration in eqs. (5) and (6) is performed by using the atomic partitioning method. ${ }^{28)}$ For the radial integration, we introduce the following variable transformation:

$$
\begin{aligned}
r & =R_{0}-\frac{1}{\beta} \log \left[\left(1+e^{\beta R_{0}}\right)^{1-a}-1\right], \\
a & =\frac{1}{2}+\frac{1}{2} x \\
x & =-\cos (\pi z) .
\end{aligned}
$$

Here, $R_{0}$ and $\beta$ are the parameters controlling the grid distribution around the atoms. $R_{0}=1$ and $\beta=1$ are appropriate for most applications. We then apply the Gauss-Chebyshev quadrature method to the variable $z$. For the angular integration, we use the spherical quadrature method. ${ }^{31-34)}$ By using the solution of eq. (4), we obtain $n_{e}^{\sigma}(\boldsymbol{r})$ by eq. (2). Then, $v_{e s}(\boldsymbol{r})$ and $v_{x c}^{\sigma}(\boldsymbol{r})$ are constructed from $n_{n}(\boldsymbol{r})$ and $n_{e}^{\sigma}(\boldsymbol{r})$. Subsequently, $v_{e s}(\boldsymbol{r})$ and $v_{x c}^{\sigma}(\boldsymbol{r})$ are used in the next iteration of the selfconsistent calculations.

Next, we describe the method to construct $v_{e s}(\boldsymbol{r})$ from $n_{n}(\boldsymbol{r})$ and $n_{e}(\boldsymbol{r})$. The calculations of the electrostatic potential due to $n_{n}(\boldsymbol{r})$ are straightforward. To calculate the electrostatic potential due to $n_{e}(\boldsymbol{r})$, we decompose $n_{e}(\boldsymbol{r})$ into the superpositional part and the residual part. The first step is to construct the superpositional part, $n_{e}^{s}(\boldsymbol{r})$. In the present study, the superpositional part is constructed in the following way.

$$
\begin{aligned}
n_{e}^{s}(\boldsymbol{r}) & =\sum_{a u} \rho_{e a}^{s}\left(\boldsymbol{r}-\boldsymbol{d}_{a}-\boldsymbol{R}_{u}\right), \\
\rho_{e a}^{s}(\boldsymbol{r}) & =\sum_{p \in a} f_{p}\left|\chi_{p}(\boldsymbol{r})\right|^{2} .
\end{aligned}
$$


Here, $f_{p}$ is the spherically averaged occupation number of the $p$-th atomic orbital belonging to the $a$-th atom. One way to obtain $f_{p}$ is to use the results of the Mulliken population analysis. Another way is to fit $n_{e}(\boldsymbol{r})$ by $n_{e}^{s}(\boldsymbol{r})$, regarding $f_{p}$ as fitting parameters. We have confirmed that both methods give good results. In the present study, we use $f_{p}$ obtained by the Mulliken population analysis. By using the spherical symmetry of $\rho_{e a}^{s}(\boldsymbol{r})$, the electrostatic potential due to $n_{e}^{s}(\boldsymbol{r})$ is calculated with a very high accuracy. The next step is to partition the residual part,

$$
n_{e}^{r}(\boldsymbol{r})=n_{e}(\boldsymbol{r})-n_{e}^{s}(\boldsymbol{r})
$$

into the atoms, by using the atomic partitioning method again. ${ }^{28)}$

$$
n_{e}^{r}(\boldsymbol{r})=\sum_{a u} \rho_{e a}^{r}\left(\boldsymbol{r}-\boldsymbol{d}_{a}-\boldsymbol{R}_{u}\right)
$$

Furthermore, these partitioned charges are decomposed into spherical harmonics components:

$$
\rho_{e a}^{r}(\boldsymbol{r})=\sum_{l m} \rho_{l m}(r) Y_{l m}(\theta, \phi) .
$$

We then solve the Poisson equations for these charges by using the method of ref. 30. Finally, the potentials due to all $l m$ components are reassembled to construct the electrostatic potential due to $n_{e}^{r}(\boldsymbol{r})$; we usually calculate the potentials up to $l=8$. As a result, $v_{e s}(\boldsymbol{r})$ is decomposed as follows:

$$
v_{e s}(\boldsymbol{r})=v_{e s}^{s}(\boldsymbol{r})+v_{e s}^{r}(\boldsymbol{r}) .
$$

Here, $v_{e s}^{s}(\boldsymbol{r})$ is the electrostatic potential due to $n_{n}(\boldsymbol{r}), n_{e}^{s}(\boldsymbol{r})$, and the associated spherical Ewald charges, which are used for the fast convergence of the lattice sum. ${ }^{35)}$ On the other hand, $v_{e s}^{r}(\boldsymbol{r})$ is the electrostatic potential due to $n_{e}^{r}(\boldsymbol{r})$ and the associated Ewald charges including nonspherical terms.

Finally, we describe the method to calculate the total energy. The total energy per unit cell, $E_{t}$, is written as

$$
E_{t}=E_{k}+E_{e s}+E_{x c} .
$$

Here, $E_{k}, E_{e s}$, and $E_{x c}$ represent the kinetic, the electrostatic, and the exchange-correlation energy, respectively. Before giving the explicit form of these energies, it should be noted that, because of the non uniqueness of the partitioning of $n_{e}(\boldsymbol{r})$ into the atomic components, the Kohn-Sham eigenvalues in solids are meaningful only if their relative values are concerned. ${ }^{36,37)}$ This is due to the conditional nature of the convergence of the lattice sum appearing in the calculations of $v_{e s}(\boldsymbol{r})$. In other words, the average potential in the solid is influenced by the details of the charge distribution on the surface. The explicit form of the energies therefore must be independent of the 
constant shift of $v_{e s}(\boldsymbol{r})$. First, $E_{k}$ is given by

$$
\begin{aligned}
E_{k} & =\frac{1}{N} \sum_{n \boldsymbol{k}_{\sigma}} f_{n \boldsymbol{k}^{\varepsilon_{n}}}^{\sigma} \boldsymbol{k}^{-} \frac{1}{N} \int_{\Omega} v_{e s}(\boldsymbol{r}) n_{e}(\boldsymbol{r}) \mathrm{d} \boldsymbol{r}-\frac{1}{N} \sum_{\sigma} \int_{\Omega} v_{x c}^{\sigma}(\boldsymbol{r}) n_{e}^{\sigma}(\boldsymbol{r}) \mathrm{d} \boldsymbol{r} \\
& =\frac{1}{N} \sum_{n \boldsymbol{k}_{\sigma}} f_{n \boldsymbol{k}^{\sigma}}^{\sigma} \varepsilon_{n \boldsymbol{k}}^{\sigma}-\sum_{a} \int_{\Omega} v_{e s}(\boldsymbol{r})\left[\rho_{e a}^{s}\left(\boldsymbol{r}-\boldsymbol{d}_{a}\right)+\rho_{e a}^{r}\left(\boldsymbol{r}-\boldsymbol{d}_{a}\right)\right] \mathrm{d} \boldsymbol{r}-\sum_{\sigma} \int_{\omega} v_{x c}^{\sigma}(\boldsymbol{r}) n_{e}^{\sigma}(\boldsymbol{r}) \mathrm{d} \boldsymbol{r}
\end{aligned}
$$

Here, the integrals over the whole solid are indicated by $\Omega$. On the other hand, the integrals over the unit cell are indicated by $\omega$, as in eqs. (5) and (6). In the above derivation, we use the periodicity of $v_{e s}(\boldsymbol{r})$ and $v_{x c}^{\sigma}(\boldsymbol{r})$. It should be noted that $E_{k}$ does not depend on the constant shift of $v_{e s}(\boldsymbol{r})$; although such a shift results in the changes in the first and the second terms in the right hand of eq. (14), the two changes are completely cancelled out. Next, $E_{e s}$ is given by

$$
\begin{aligned}
E_{e s} & =\frac{1}{2 N} \int_{\Omega}^{\prime} v_{e s}(\boldsymbol{r})\left[n_{e}(\boldsymbol{r})-n_{n}(\boldsymbol{r})\right] \mathrm{d} \boldsymbol{r} \\
& =\frac{1}{2} \sum_{a} \int_{\Omega}^{\prime} v_{e s}(\boldsymbol{r})\left[\rho_{e a}^{s}\left(\boldsymbol{r}-\boldsymbol{d}_{a}\right)+\rho_{e a}^{r}\left(\boldsymbol{r}-\boldsymbol{d}_{a}\right)-\rho_{n a}\left(\boldsymbol{r}-\boldsymbol{d}_{a}\right)\right] \mathrm{d} \boldsymbol{r} .
\end{aligned}
$$

Here $\rho_{n a}(\boldsymbol{r})$, and therefore $n_{n}(\boldsymbol{r})$, is represented by using the atomic number with the delta function:

$$
\begin{gathered}
n_{n}(\boldsymbol{r})=\sum_{a u} \rho_{n a}\left(\boldsymbol{r}-\boldsymbol{d}_{a}-\boldsymbol{R}_{u}\right), \\
\rho_{n a}\left(\boldsymbol{r}-\boldsymbol{d}_{a}\right)=Z_{a} \delta\left(\boldsymbol{r}-\boldsymbol{d}_{a}\right) .
\end{gathered}
$$

Here $Z_{a}$ and $\boldsymbol{d}_{a}$ represent the atomic number and the position vector of the $a$-th atom in the unit cell, respectively. In eq. (15), the prime of the integrals indicates the exclusion of the nuclear self-interaction energies. It is obvious that $E_{e s}$ also does not depend on the constant shift of $v_{e s}(\boldsymbol{r})$ because of the charge neutrality. Finally, $E_{x c}$ is given by

$$
\begin{aligned}
E_{x c} & =\frac{1}{N} \sum_{\sigma} \int_{\Omega} \epsilon_{x c}^{\sigma}(\boldsymbol{r}) n_{e}^{\sigma}(\boldsymbol{r}) \mathrm{d} \boldsymbol{r} \\
& =\sum_{\sigma} \int_{\omega} \epsilon_{x c}^{\sigma}(\boldsymbol{r}) n_{e}^{\sigma}(\boldsymbol{r}) \mathrm{d} \boldsymbol{r} .
\end{aligned}
$$

Here, $\epsilon_{x c}^{\sigma}(\boldsymbol{r})$ is the exchange-correlation energy density. In the present study, we also use the PerdewZunger parameterization of Ceperley and Alder results as $\epsilon_{x c}^{\sigma}(\boldsymbol{r}) \cdot{ }^{41,42)}$ For the efficient calculations of $E_{t}$, it is essential to divide the calculations into two parts. ${ }^{11,26)}$ One is the superpositional part calculated with a very high accuracy. Another is the residual part requiring the three-dimensional numerical integration. By using the spherical symmetry of $\rho_{e a}^{s}\left(\boldsymbol{r}-\boldsymbol{d}_{a}\right), \rho_{n a}\left(\boldsymbol{r}-\boldsymbol{d}_{a}\right)$, and the associated spherical Ewald charges, one can calculate the superposional part of the total energy, 
$E_{t}^{s}$, avoiding the three-dimensional numerical integration. We only necessary to calculate two-center integrals for the electrostatic energies between the spherical charges.

$$
E_{t}^{s}=\frac{1}{N} \sum_{n \boldsymbol{k}_{\sigma}} f_{n \boldsymbol{k}}^{\sigma} \boldsymbol{k}_{n \boldsymbol{k}}^{\sigma}-\frac{1}{2} \sum_{a} \int_{\Omega}^{\prime} v_{e s}^{s}(\boldsymbol{r})\left[\rho_{e a}^{s}\left(\boldsymbol{r}-\boldsymbol{d}_{a}\right)+\rho_{n a}\left(\boldsymbol{r}-\boldsymbol{d}_{a}\right)\right] \mathrm{d} \boldsymbol{r} .
$$

As a result, the three-dimensional numerical integration is required only for the residual part, $E_{t}^{r}$.

$$
E_{t}^{r}=-\frac{1}{2} \int_{\omega}\left\{v_{e s}^{r}(\boldsymbol{r})\left[n_{e}(\boldsymbol{r})+n_{n}(\boldsymbol{r})\right]+v_{e s}^{s}(\boldsymbol{r}) n_{e}^{r}(\boldsymbol{r})\right\} \mathrm{d} \boldsymbol{r}+E_{x c}-\sum_{\sigma} \int_{\omega} v_{x c}^{\sigma}(\boldsymbol{r}) n_{e}^{\sigma}(\boldsymbol{r}) \mathrm{d} \boldsymbol{r} .
$$

In the calculations of the atomization energies, we further reduce the numerical error of $E_{t}^{r}$ by subtracting the total energy of the isolated atoms by calculating it with the same numerical grids. ${ }^{16)}$

\section{§3. Application to Graphite and Hexagonal Boron Nitride}

In this section, we present the results of the calculations of the interatomic distances, the atomization energies, the elastic constants $C_{11}+C_{12}$, and the band structures of graphite and hexagonal boron nitride $(h$-BN).

We use two kinds of basis sets for graphite. ${ }^{16,22)}$ One is the double basis (DB) comprised of $1 s$, $2 s$, and $2 p$ orbitals of a neutral $\mathrm{C}$ atom and $2 s$ and $2 p$ orbitals of a $\mathrm{C}^{2+}$ ion. Another is DB plus one $d$ orbital (DBD) including one $3 d$ orbital of a $\mathrm{C}^{2+}$ ion as a polarization function. Also, we use DBD for $\mathrm{B}$ and $\mathrm{N}$ for the calculations of $h$-BN. Furthermore, all the results presented here are obtained by using 24 radial and 86 spherical mesh points per atom for the three-dimensional integration.

The sampling in BZ is performed by using two methods. ${ }^{38,39)}$ One is the good-lattice-point method (GLPM) and another is the special-point method (SPM). Since GLPM gives good results for the integration of periodic functions, this method is suitable for the sampling in BZ. GLPM in three dimension, however, requires at least 185 sampling points. This results in very heavy calculational costs. On the other hand, SPM needs only several sampling points, three for graphite and $h$-BN. Since the reliability of this method is restricted to non metals, we must check its applicability to graphite, which is a semimetal. Then, we apply GLPM and SPM to the structure optimization of graphite with DB to confirm the applicability of SPM. Next, the structure optimization is performed for graphite and $h$-BN by SPM with DBD. Finally, using the optimized structures, we perform the self-consistent-field calculations by GLPM with DBD and calculate the band structures of graphite and $h$-BN.

Table I shows the interatomic distances, the atomization energies, and the elastic constants $C_{11}+C_{12}$ with the experimental results; we calculate $C_{11}+C_{12}$ by using a formula in ref. 49 . The used basis sets and the BZ sampling method are also shown. Since the nonlocal corrections to the LSDA energies may be essential to the treatment of the weak van der Waals binding between the layers, we optimize the intralayer interatomic distances only, fixing the interlayer distances to the experimental values: $3.40 \AA$ for graphite and $3.33 \AA$ for $h$-BN. 
First, it is found that the results for graphite by SPM with DB are good as same as those by GLPM with DB. That is, although graphite is a semimetal, the BZ sampling by SPM can give the reliable results. Also, this ensures the applicability of SPM to $h$-BN because it is an insulator. Then, we apply SPM with DBD to the structure optimization of graphite and $h$-BN. The results are also shown in Table I. For graphite, the only change due to the use of the better quality of basis set is the increase in the atomization energies, while the interatomic distances and $C_{11}+C_{12}$ are almost unchanged. The atomization energy is overestimated comparing with the experimental one, as is attributable to the use of LSDA, while the theoretical interatomic distances and $C_{11}+C_{12}$ are in good agreement with the experimental values. These tendencies are also found in the results of $h$-BN.

We now refer to the numerical error. The numerical error in the interatomic distances is found to be less than $0.01 \AA$ while that in the atomization energies is found to be about $0.1 \mathrm{eV} /$ atom. The error in the atomization energies primarily arises from the radial and the spherical integration while the error associated with the BZ sampling is less than $0.05 \mathrm{eV} /$ atom. Considering this, one reason for the success of the structure optimization is the systematic occurrence of the errors in the atomization energies around the optimized structures.

By using the optimized structures obtained by SPM with DBD, we perform the self-consistentfield calculations of graphite and $h$-BN, and then calculate the band structures. The results of the calculations are shown in Fig. 1 for graphite and in Fig. 2 for $h$-BN. Also, several eigenvalues at $\Gamma$ point are given in Table II for graphite and in Table III for $h$-BN with available results of experiments and other calculations. The overall agreement with the other calculations is good for both graphite and $h$-BN. Finally, it should be stressed that the number of the basis functions in the present study, 14 per atom, is very small in comparison with that used in the APW or PP-PW calculations, which requires more than 100 basis functions per atom. This is an advantage of the present method.

\section{$\S 4$. Conclusions}

In the present study, we have shown that the full-potential linear-combination-of-atomic-orbitals method developed in the field of the molecular science is also useful for studying both the structural and electronic properties of solids. In particular, the efficient method of the total-energy calculations, the atomic partitioning method of the three-dimensional numerical integration, and the method of solving the Poisson equation are successfully used in the present method. By using the present method, we study the structural and electronic properties of graphite and hexagonal boron nitride. The agreement of the calculated interatomic distances with the experimental ones is very good. Also, the atomization energies and the elastic constants are properly reproduced. 


\section{References}

1) P. Hohenberg and W. Kohn: Phys. Rev. 136 (1964) B864.

2) W. Kohn and L. J. Sham: Phys. Rev. 140 (1965) A1133.

3) J. R. Chelikowsky and M. L. Cohen: Handbook on Semiconductors Vol.1 (North-Holland, Amsterdam, 1992).

4) A. J. Freeman, C. L. Fu, S. Ohnishi, and M. Weinert: Polarized Electrons in Surface Physics (World Scientific Publishing Co., Singapore, 1985).

5) K. Klemradt, B. Drittler, T. Hoshino, R. Zeller, P. H. Dederichs, and N. Stefanou: Phys. Rev. B 43 (1991) 9487.

6) O. K. Andersen, O. Jepsen, and D. Glötzel: Highlights in Condensed Matter Theory (North-Holland, New York, 1985).

7) S. C. Erwin, M. R. Pederson, and W. E. Pickett: Phys. Rev. B 41 (1990) 10437.

8) G. te Velde and E. J. Baerends: Phys. Rev. B 44 (1991) 7888.

9) A. Zupan and M. Causà: Int. J. Quantum Chem. 56 (1995) 337.

10) D. E. Ellis and G. S. Painter: Phys. Rev. B 2 (1970) 2887.

11) E. J. Baerends, D. E. Ellis, and P. Ros: Chem. Phys. 2 (1973) 41.

12) E. J. Baerends and P. Ros: Chem. Phys. 2 (1973) 52.

13) H. Sambe and R. H. Felton: J. Chem. Phys. 62 (1975) 1122.

14) H. Adachi, M. Tukada, and C. Satoko: J. Phys. Soc. Jpn. 45 (1978) 875.

15) B. Delley and D. E. Ellis: J. Chem. Phys. 76 (1982) 1949.

16) B. Delly, D. E. Ellis, A. J. Freeman, E. J. Baerends, and D. Post: Phys. Rev. B 27 (1983) 2132.

17) B. I. Dunlap, J. W. D. Connolly, and J. R. Sabin: J. Chem. Phys. 71 (1979) 3396.

18) B. I. Dunlap, J. W. D. Connolly, and J. R. Sabin: J. Chem. Phys. 71 (1979) 4993.

19) C. Satoko: Chem. Phys. Lett. 83 (1981) 111.

20) C. Satoko: Phys. Rev. B 30 (1984) 1754.

21) L. Versluis and T. Ziegler: J. Chem. Phys. 88 (1988) 322.

22) B. Delley: J. Chem. Phys. 92 (1990) 508.

23) B. Delley: J. Chem. Phys. 94 (1991) 7245.

24) J. Andzelm and E. Wimmer: J. Chem. Phys. 96 (1992) 1280.

25) K. Kobayashi, K. Tago, and N. Kurita: Phys. Rev. A 53 (1996) 1903.

26) T. Ziegler and A. Rauk: Theoret. Chim. Acta 46 (1977) 1.

27) P. M. Boerrigter, G. te Velde, and E. J. Baerends: Int. J. Quantum Chem. 33 (1988) 87.

28) A. D. Becke: J. Chem. Phys. 88 (1988) 2547.

29) F. W. Averill and G. S. Painter: Phys. Rev. B 39 (1989) 8115.

30) A. D. Becke and R. M. Dickson: J. Chem. Phys. 89 (1988) 2993.

31) V. I. Lebedev: Zh. Vychisl. Mat. Mat. Fiz. 15 (1975) 48.

32) V. I. Lebedev: Zh. Vychisl. Mat. Mat. Fiz. 16 (1976) 293.

33) V. I. Lebedev: Sibirsk. Mat. Zh. 18 (1977) 132.

34) S. I. Konyaev: Mat. Zametki 25 (1979) 629.

35) B. Delley: J. Phys. Chem. 100 (1996) 6107.

36) J. Ihm and M. L. Cohen: Phys. Rev. B 21 (1980) 3754.

37) L. Kleinman: Phys. Rev. B 24 (1981) 7412.

38) L.-K. Hua and Y. Wang: Applications of Number Theory to Numerical Analysis (Springer-Verlag, Berlin, 1981).

39) D. J. Chadi and M. L. Cohen: Phys. Rev. B 8 (1973) 5747. 
40) A. Zunger and A. J. Freeman: Phys. Rev. B 15 (1977) 4716.

41) J. P. Perdew and A. Zunger: Phys. Rev. B 23 (1981) 5048.

42) D. M. Ceperley and B. J. Alder: Phys. Rev. lett. 45 566(1980) 566.

43) R. W. G. Wyckoff: Crystal Structures (Interscience, New York, 1965).

44) Numerical Data and Functional Relationships in Science and Technology, edited by O. Madelung, M. Schulz, and H. Weiss, Landolt-Börnstein, New Series, (Springer-Verlag, Berlin, 1984).

45) W. Eberhardt, J. T. McGovern, E. W. Plummer, and J. E. Fischer: Phys. Rev. Lett. 44 (1980) 200.

46) A. R. Law, J. J. Barry, and H. P. Hughes: Phys. Rev. B 28 (1983) 5332.

47) A. Bianconi, S. B. M. Hagström, and R. Z. Bachrach: Phys. Rev. B 16 (1977) 5543.

48) J.-C. Charlier, X. Gonze, and J.-P. Michenaud: Phys. Rev. B 43 (1991) 4579.

49) H. J. F. Jansen and A. J. Freeman: Phys. Rev. B 35 (1987) 8207.

50) N. A. W. Holtzwarth, S. G. Louie, and S. Rabii: Phys. Rev. B 26 (1982) 5382.

51) R. C. Tatar and S. Rabii: Phys. Rev. B 25 (1982) 4126.

52) A. Nagashima, N. Tejima, Y. Gamou, T. Kawai, and C. Oshima: Phys. Rev. B 51 (1995) 4606.

$53)$ X. Blase, A. Rubio, S. G. Louie, and M. L. Cohen: Phys. Rev. B 51 (1995) 6868.

54) A. Catellani, M. Posternak, A. Baldereschi, H. J. F. Jansen, and A. J. Freeman: Phys. Rev. B 32 (1985) 6997.

55) Y.-N. Xu and W. Y Ching: Phys. Rev. B 44 (1991) 7787. 


\section{Figure Captions}

Fig. 1. Band structure of graphite.

Fig. 2. Band structure of hexagonal BN. 
Table I. Interatomic distance in $\AA$, atomization energy in $\mathrm{eV} /$ atom, and $C_{11}+C_{12}$ in GPa of graphite and hexagonal $\mathrm{BN}$.

\begin{tabular}{|c|c|c|c|c|c|c|c|c|}
\hline \multirow[t]{2}{*}{ Solid } & \multicolumn{2}{|c|}{$\begin{array}{l}\text { Interatomic } \\
\text { distance }^{a)}\end{array}$} & \multicolumn{2}{|c|}{$\begin{array}{c}\text { Atomization } \\
\text { energy }^{\mathrm{b})}\end{array}$} & \multicolumn{2}{|c|}{$C_{11}+C_{12}^{\mathrm{c})}$} & \multirow[t]{2}{*}{$\begin{array}{c}\text { Basis } \\
\text { set }\end{array}$} & \multirow[t]{2}{*}{$\begin{array}{c}\text { BZ } \\
\text { sampling }\end{array}$} \\
\hline & Expt. & This work & Expt. & This work & Expt. & This work & & \\
\hline \multirow[t]{3}{*}{ Graphite } & 1.42 & 1.42 & 7.41 & 8.68 & 1240 & 1208 & DB & GLP \\
\hline & & 1.42 & & 8.71 & & 1221 & DB & $\mathrm{SP}$ \\
\hline & & 1.41 & & 8.97 & & 1214 & $\mathrm{DBD}$ & SP \\
\hline Hexagonal BN & 1.45 & 1.44 & 6.63 & 8.03 & 951 & 1164 & DBD & SP \\
\hline
\end{tabular}

a) The experimental interatomic distances are taken from ref. 43.

b) The experimental atomization energies are calculated by standard heat of formation at $298.15 \mathrm{~K}$.

c) The experimental $C_{11}+C_{12}$ are taken from ref. 44 . 
Table II. One-electron energies of graphite at $\Gamma$ point in $\mathrm{eV}$.

\begin{tabular}{|c|c|c|c|c|c|c|c|c|}
\hline & \multicolumn{3}{|c|}{ Expt. } & \multicolumn{5}{|c|}{ Theoretical } \\
\hline & Ref. 45 & Ref. 46 & Ref. 47 & This work & Ref. 48 & Ref. 49 & Ref. 50 & Ref. 51 \\
\hline \multirow[t]{2}{*}{ Bottom $\sigma$} & -20.6 & & & -19.5 & -20.1 & -19.6 & -20.8 & -19.5 \\
\hline & & & & -19.2 & -19.8 & -19.3 & -20.5 & -19.2 \\
\hline \multirow[t]{2}{*}{ Bottom $\pi$} & -8.1 & -8.5 & & -8.5 & -8.9 & -8.7 & -9.1 & -8.2 \\
\hline & -7.2 & -6.6 & -5.7 & -6.6 & -6.8 & -6.7 & -7.1 & -6.5 \\
\hline \multirow[t]{2}{*}{ Top $\sigma$} & -4.6 & -5.5 & & -3.2 & -3.5 & -4.6 & -3.4 & -4.3 \\
\hline & & & & -3.1 & -3.4 & -4.6 & -3.3 & -4.3 \\
\hline \multirow[t]{3}{*}{ Unoccupied $\sigma^{*}$} & & & & 3.9 & 3.7 & 3.8 & 3.7 & 7.1 \\
\hline & 6.9 & & & 8.2 & 7.9 & 8.3 & 9.0 & 7.3 \\
\hline & & & & 8.2 & 7.9 & 8.4 & 9.3 & 7.3 \\
\hline
\end{tabular}


Table III. One-electron energies of hexagonal BN at $\Gamma$ point in $\mathrm{eV}$.

\begin{tabular}{|c|c|c|c|c|c|}
\hline & \multirow{2}{*}{$\begin{array}{c}\text { Expt. } \\
\text { Ref. } 52\end{array}$} & \multicolumn{4}{|c|}{ Theoretical } \\
\hline & & This work & Ref. 53 & Ref. 54 & Ref. 55 \\
\hline \multirow[t]{2}{*}{ Bottom $\sigma$} & & -17.8 & -17.9 & -19.3 & -18.7 \\
\hline & & -17.5 & -17.7 & -18.9 & -18.2 \\
\hline \multirow[t]{2}{*}{ Bottom $\pi$} & -5.7 & -6.4 & -6.3 & -6.7 & -6.7 \\
\hline & & -4.3 & -4.1 & -4.2 & -4.5 \\
\hline \multirow[t]{2}{*}{ Top $\sigma$} & -0.9 & -1.6 & -1.5 & -2.0 & -2.5 \\
\hline & & -1.5 & -1.3 & -1.7 & -2.4 \\
\hline \multirow[t]{3}{*}{ Unoccupied $\sigma^{*}$} & & 5.1 & 4.6 & 4.3 & 6.3 \\
\hline & & 10.0 & 10.1 & 10.6 & 10.1 \\
\hline & & 11.3 & 11.2 & 11.0 & 10.6 \\
\hline
\end{tabular}




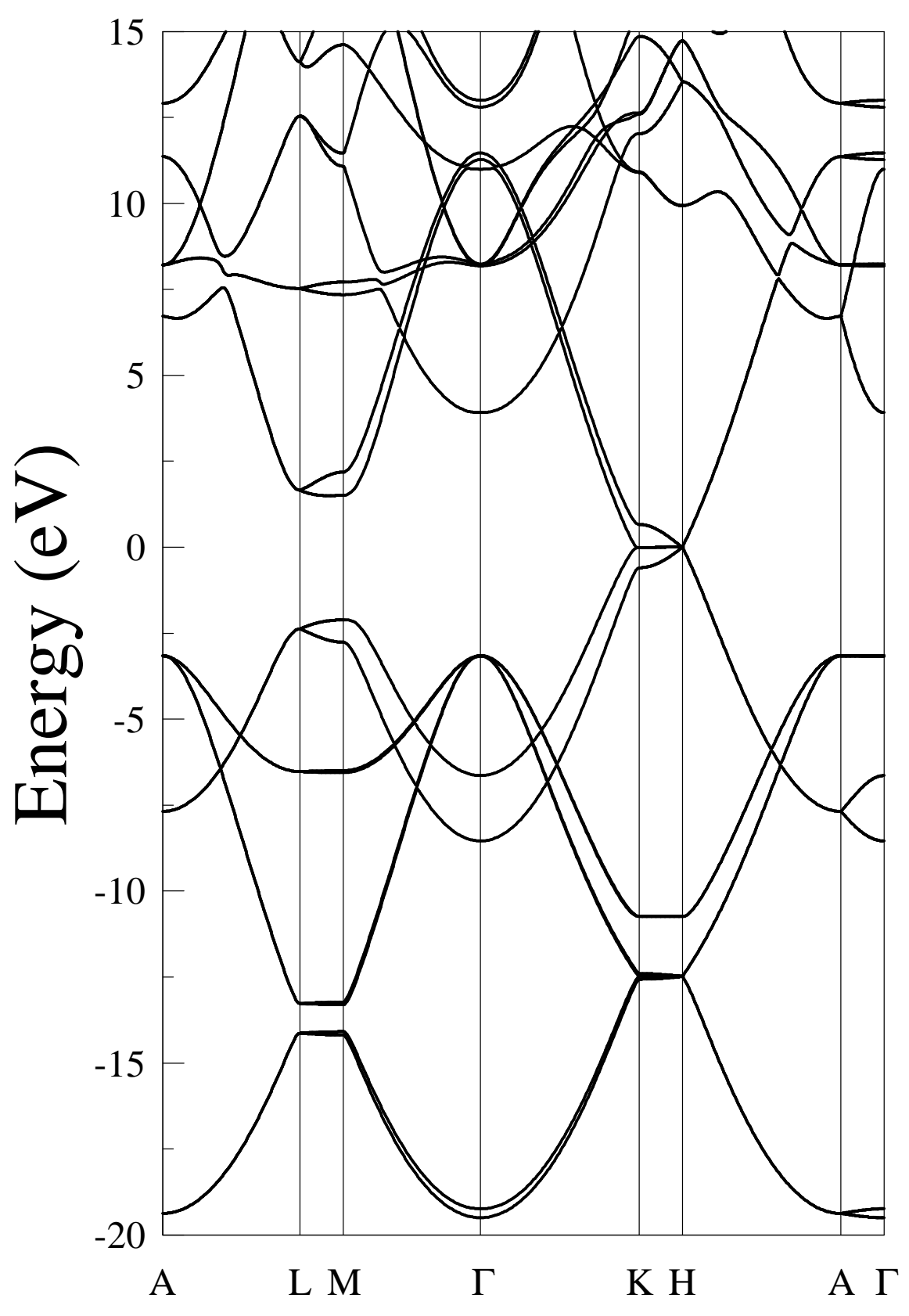

Fig. 1 


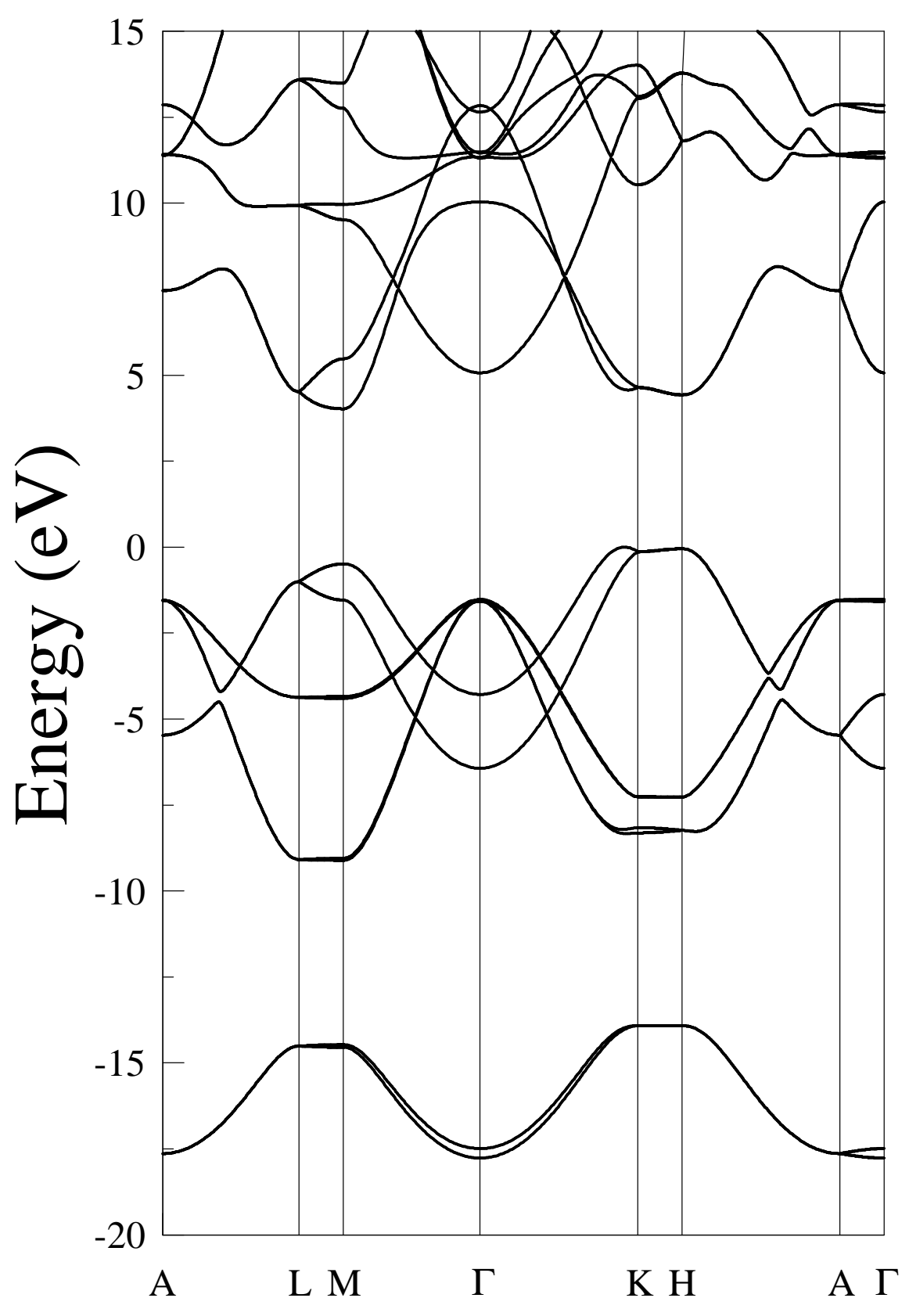

Fig. 2 